\title{
Ant colony optimization based energy efficiency for improving opportunistic routing in multimedia wireless mesh network
}

\author{
S. Harikishore, V. Sumalatha \\ ${ }^{1}$ Department of Electronics and Communication Engineering, JNTUA College of Engineering, Anantapur, India
}

\begin{tabular}{l} 
Article Info \\
\hline Article history: \\
Received Dec 20, 2018 \\
Revised Mar 17, 2019 \\
Accepted Apr 11, 2019 \\
\hline
\end{tabular}

\section{Keywords:}

Ant colony optimization Energy efficiency Wireless mesh network opportunistic routing

\begin{abstract}
Opportunistic Routing (OR) is developing as a favourable prototype to diminish performance deprivation in Wireless Mesh Networks (WMNs) owing to changing channel conditions and link breakages. When a flow of data is forwarded towards their destination, intermediate forwarders can attune the information of the route carried by the nodes. However, OR does not solve these problems such as routing efficiency and Energy Consumption. If the necessary energy is not presented, the packet is rejected and the delay occurs in the network. To overcome these problems, an Ant Colony Optimization based Energy Efficiency for improving opportunistic routing in Multimedia WMN (ACO-EE) is proposed. In this scheme, we develop the optimal energy strategy based on optimal transmission distance and remaining energy computation is saving node energy and enhancing the network lifetime. The ant colony optimization based route formation is to improve both the energy efficiency and opportunistic routing efficiency in WMN.Simulation results show that ACE-EE can effectively reduce the energy utilization of nodes and extend the network lifetime.
\end{abstract}

Copyright $(0) 2019$ Institute of Advanced Engineering and Science. All rights reserved.

\section{Corresponding Author:}

S. Harikishore,

Department of Electronics and Communication Engineering,

JNTUA College of Engineering,

Anantapur, Andhra Pradesh, India.

Email: harikishorephd@gmail.com

\section{INTRODUCTION}

Energy efficiency is the essential features to consider when planning protocols for WMNs. Recently, opportunistic routing techniques are introduced to helpfulness in supporting the transmission between nodes, and its facility to develop the capability of the wireless broadband via multi-hop frontward proficiency [1]. Normally, wireless multimedia networks should need reliable communication and energy efficiency while keeping suitable Quality of Service (QoS). In order to obtain the least energy utilization throughout data communication in the network, many energy efficiency concept is introduced. Though, the optimal energy approach does not clearly proceeds the remaining energy of forwarding nodes in WMN. The ant colony optimization (ACO) algorithm is motivated by the ants' food examine behaviour in nature. The ACO algorithm can diminish time of search paths by graphs [2].

In this paper, we intend an energy-efficient routing for multimedia WMN, explicitly; Ant Colony Optimization based Energy Efficiency for improving opportunistic routing in Multimedia WMN.

The main contributions of this paper include the following.

a) We describe the optimal energy strategy based on the optimal transmissionDistance and remaining energy.

b) We select the Forwarding Candidate Set (FCS) based on the node connectivity, node communication range. 
c) We introduce the ACO algorithm to find the efficient route and transmitting the data via forwarder candidate nodes.

d) The ACO algorithm selects the Forwarder Candidate (FC) by node pheromone value. This pheromone value is computed by optimal energy, hop count and data communication time.Thus, improve the routing efficiency and network lifetime.

In the literature, several energy-aware protocols have been proposed for the wireless network. Again, there are several routing protocols proposed for WSN, in which the main focus is on reliable data delivery.

Energy-Efficient SINR-Based Routing [3] introduced an energy-efficient algorithm for certain bandwidth restraints of incoming flows. This scheme mainly used to reduce energy consumption in the network. Localized Energy-Aware Restricted Neighborhood routing scheme [4] the node chooses the neighbor within a constrained neighborhood that has the prevalent energy mileage as the next hop. It provides the guarantee of energy efficiency routes in the multi-hop wireless network. Forward-aware factor based energy balanced routing mechanism [5] for enhancing energy efficiency in the network. In this scheme, the hop is elected by the density of forwarding energy and link weight. This scheme provides robustness topology, minimizes the link breakage problem, QoS routing. An efficient Power-Aware Routing [6] is proposed for improving the network lifetime. In this scheme, the next hop is elected by remaining battery power and expected energy. This scheme takes the route has the highest data packet capacity at the minimum remaining packet communication capacity. It minimizes the average delay, energy utilization during heavy network load situation.

Swarm intelligence algorithm [7] introduced for reliable and energy efficient routing in the wireless network. This algorithm can accommodate to the network topology, improve the network lifespan and minimize the energy utilization in the network. Jumper Firefly an optimization technique [8] mainly concentrates on resource management problem regarding energy that increases the lifetime of the network. This technique can extend the communication range of nodes towards the void area in the network. An energy efficient multipath routing [9] is using the fitness function to discover the optimal path from source to destination. The optimal route is selected by node energy and route distance for enhancing the lifespan of the network.

Energy Efficient Relay Selection (EERS) [10] for enhancing the energy efficiency in Cooperative Wireless Multimedia Networks. In EERS, the power-allotment process to optimize the cooperative communication for source and relay nodes to minimize the energy utilization, though preserving the essential QoS, and cooperative communication in terms of energy efficiency. However, EERS cannot provide robust cooperative communication and it not able to improve the network lifespan. In addition, EERS does not work perform when increasing the network traffic load. Energy-efficient cooperative multicasting scheme [11] is introduced for minimizing the cooperative quantity of energy utilization in the network. In this scheme, the relay agent is selected based on their coverage, position and channel condition. However, this scheme increases the additional network overhead.

ACO algorithm is motivated from self-organizing behavior of ants that decrease under Swarm Intelligence [12]. ACO algorithm utilizing cooperative intelligence of artificial ants as intelligent agents is very suitable to resolve the combinative optimization problems in a completely disseminated method, thus the updated ACO algorithm to resolve Delay Restraint, Maximum Energy Remaining Ratio, QoS routing problem. ACO based routing considers about optimization techniques for example Weighted Compressive Data Aggregation (WCDA), Cluster-based Weighted Compressive Data Aggregation (CWCDA) and ACO. WCDA and CWCDA algorithm is used for reducing energy consumption in the network [13]. The ACO based QoS routing algorithm (ACO-QoSR) for finding best paths that satisfied with the QoS necessities. ACO-QoSR algorithm is the tradeoff among a sure guaranteed QoS requests and suitable computational difficulty [14].

\section{ACO BASED ENERGY EFFICIENCY FOR IMPROVING OPPORTUNISTIC ROUTING IN MULTIMEDIA WMN}

The main aim of this scheme is to improve an energy-efficient opportunistic routing in multimedia WMN. Here, we conceive a number of nodes and every node has enough neighbor nodes represents $\mathrm{H}$ in a multi-hop WMN. This network built by an associated graph $\mathrm{AG}(\mathrm{V}, \mathrm{E})$. Where $\mathrm{V}$ represents the nodes and $\mathrm{E}$ represents the associated link among nodes.

\subsection{Energy Model}

In this scheme, we discuss to a simplified energy model of radiocommunication as it is used in [15]. The energy utilization to transmit an M-bit message can be explicit as follows 


$$
E_{\text {Trans }}=\left(E_{\text {elece }}+\varepsilon_{\text {ampl }} d^{v}\right) M
$$

The energy utilization of the receiver can be computed as follows

$$
E_{\operatorname{Re} c}=E_{\text {elece }} M
$$

Here, $E_{\text {elece }} \rightarrow$ energy utilization of node to operate the receiver or transmitter circuitry $\mathrm{E}_{\mathrm{ampl}} \rightarrow$ energy spread out in the broadcast amplifier.

$\mathrm{d} \rightarrow$ distance among receiver and transmitter

$v \rightarrow$ Path-loss exponent

\subsection{Optimal Energy Strategy}

Our aim is to design an optimal energy strategy for each relay node that ensures utilized minimum energy. The location of node $\mathrm{A}$ indicates the $\mathrm{X}_{\mathrm{A}}$ and based on energy model optimal communication distance for node $\mathrm{A}$ is computed by:

$$
O P_{d i s}=\frac{n-X_{A}}{K}
$$

The distance among node $\mathrm{A}$ to destination $\mathrm{D}$ is $\mathrm{D}_{\mathrm{A} \rightarrow \mathrm{D}}$ computed by

$$
D i s_{A \rightarrow D}=n-x_{A}=\sum_{i=1}^{k} x_{i}-x_{i-1}
$$

Where k represents the hop count from node A to the destination, $\mathrm{n}$ represents the destination location. Thus, the total consumed energy of node A to D can be computed as follows.

$$
\begin{aligned}
& C E_{A \rightarrow D}=\sum_{i=1}^{k}\left\{\left(E_{\text {elece }}+\varepsilon_{\text {ampl }}\left(x_{i}-x_{i-1}\right)^{v}\right) M\right\}+\sum_{i=1}^{k} E_{\text {elece }} M \\
& \beta=\sum_{i=1}^{k}\left\{\left(E_{\text {elece }}+\varepsilon_{\text {ampl }}\left(x_{i}-x_{i-1}\right)^{v}\right) M\right\}+\sum_{i=1}^{k} E_{\text {elece }} M
\end{aligned}
$$

Here, we can reach optimal energy by selecting optimal hops $\mathrm{K}$ to find optimal transmission distance $\mathrm{OP}_{\text {dis. }}$ Let take the node $\mathrm{A}$ is transmitting a data packet to destination, and $\mathrm{A}+\mathrm{i}$ is one of FCS of node A. If it is a minimum distance and has more remaining energy, the node $\mathrm{A}+\mathrm{i}$ can be a FC, then thenetwork can get better energy usage. The optimal energy computation is given below.

$$
O E(\mathrm{~A}+i)=d_{A+i}-d_{A}\left[\frac{1}{d_{A+i}-O P_{d i s}}+\left(\mathrm{E}_{A+i}-\beta\right)\right]
$$

Where,

$d_{A+i}-d_{A} \rightarrow$ Distance among node $\mathrm{A}$ and neighbor node $\mathrm{A}+\mathrm{i}$

$\mathrm{E}_{A+i} \rightarrow$ Remaining energy of node $\mathrm{A}+\mathrm{i}$

$\beta \rightarrow$ Energy Threshold

In this scheme, we explain how to choose the Forwarding Candidate Set and pick the Forwarder Candidate based on the ACO algorithm.

\subsection{ACO Algorithm}

In this scheme, we formed the route by ant colony optimization technique. Forward ant messages are created by the source for discoverseveral paths to the destination, and backward ants messages return 
following the traces to set up the paths. The pheromone valuespecified the particularpaths along with the quality information.

During the route discovery phase, the source node broadcasts forward ant message to the neighbor nodes and then collects the FCS that have better connectivity and within communication range. The node with high connectivity implies more neighbor nodes around the position at which the node is located [16]. While the forward ants achieve the destination, then the destination produced the backward ant message to the FCS. This backward ant message updates the pheromone value of every node.

During route selection phase, the source selects the FC nodes by the pheromone value. The pheromone value of each node can be computed based on the node optimal energy, hop count and Data Communication Time. The backward ant message format is given below.

Table 1. Backward Ant Message Format

\begin{tabular}{cccccc}
\hline $\begin{array}{c}\text { Destination } \\
\text { address }\end{array}$ & $\begin{array}{c}\text { Source } \\
\text { address }\end{array}$ & $\begin{array}{c}\text { Optimal } \\
\text { energy }\end{array}$ & $\begin{array}{c}\text { Hop } \\
\text { Count }\end{array}$ & $\begin{array}{c}\text { Data communication } \\
\text { time }\end{array}$ & $\begin{array}{c}\text { Time to } \\
\text { Live }\end{array}$ \\
\hline
\end{tabular}

The data communication time (DCT) is measured that the node was how much amount of time taken for data transmission.

$$
D C \mathrm{~T}=\text { DPack Rcvd }_{\text {time }}-\text { DPack send } \text { time }
$$

During data transmission, the Source reaches the several backward ant messages then the Source selects the FC based on the highest pheromone value for data transmission. The pheromone value for node $\mathrm{i}$ can be computed as follows.

$$
P(\mathrm{i})=\frac{\left(O E_{\mathrm{G}} H C_{\mathrm{G}} \mathrm{DCT}_{\mathrm{G}}\right) i}{\sum_{j \in F C S}\left(O E_{\mathrm{G}} H C_{\mathrm{G}} \mathrm{DCT}_{\mathrm{G}}\right)_{\mathrm{j}}}
$$

$O E_{\mathrm{G}}=$ Goodness of optimal energy

$H C_{\mathrm{G}}=$ Goodness of Hop Count

$D C T_{\mathrm{G}}=$ Goodness of Data Communication Time Figure 1.

Hence, the data reaches thedestination successfully. The flowchart for the ACO-EE is shown in

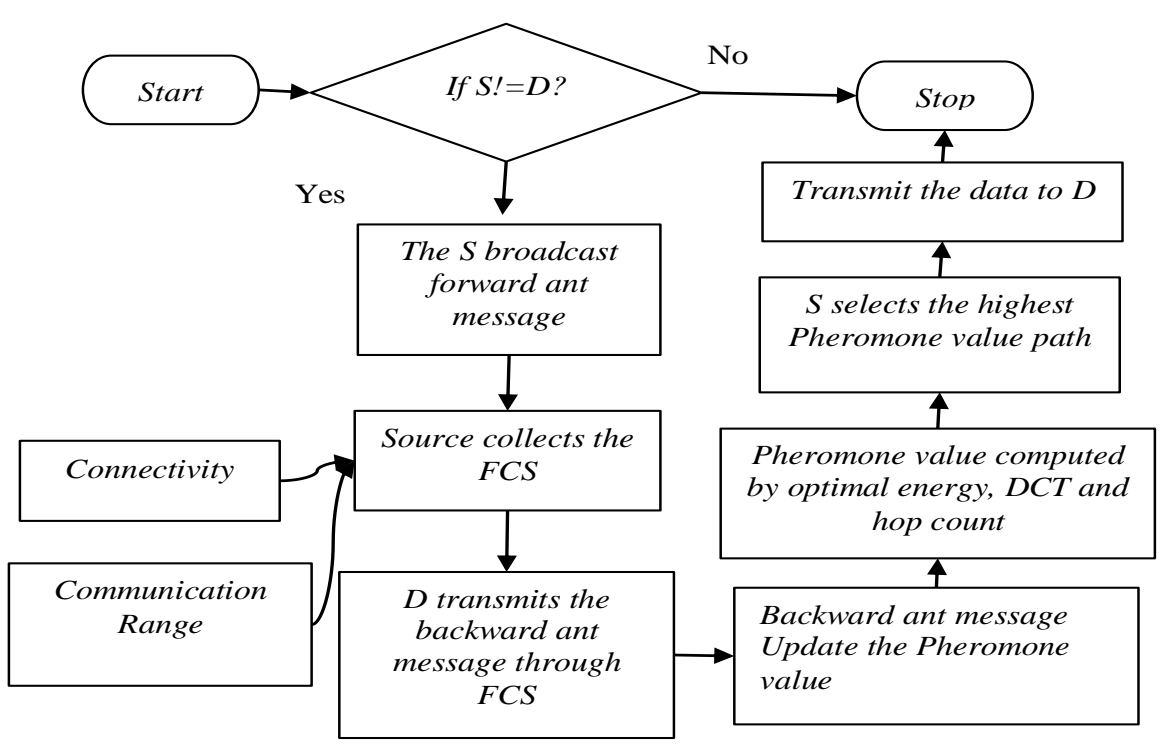

Figure 1. Flowchart of ACO-EE scheme 


\section{RESULTS AND ANALYSIS}

In this section, we investigate the performance of ACO-EE by simulation using Network Simulator ns-2.35. The simulation parameters are mentioned in Table 2. We compare it against EERS with varying simulation parameters such as received packets, lost packets, residual energy and average delay and average remaining energy are estimated in ACO-EE and EERS.

Receiving packets rate (RPR): RPR is determined as the ratio of the quantity of packets obtained by the destination to the total quantity of packets transmits by the source. The received packets rate RPRis computed using the formula 9 .

$$
R P R=\frac{\sum_{0}^{n} \operatorname{PktRcv}(n)}{\sum_{0}^{n} \operatorname{PktRcv}(n)+\sum_{0}^{n} \operatorname{Pkt} \operatorname{Lost}(n)}
$$

The Loss of packets rate LPR is computed using the formula 10.

$$
L P R=\frac{\sum_{0}^{n} P k t \operatorname{Lost}(n)}{\sum_{0}^{n} P k t R c v(n)+\sum_{0}^{n} P k t \operatorname{Lost}(n)}
$$

Delay per node is measured using the formula 11.

Table 2. Simulation Parameters of ACO-EE

\begin{tabular}{cc}
\hline Parameter & Value \\
\hline \# nodes & 50 \\
Model of Antenna & Omni Antenna \\
Type of Network Interface & WirelessPhy \\
Model of Radio Propagation & TwoRayGround \\
Simulation Area & $600 \times 600$ \\
Simulation Time & $50 \mathrm{~s}$ \\
Model of Traffic & Constant Bit Rate \\
Transmission Range & $250 \mathrm{~m}$ \\
Type of Channel & Wireless Channel \\
Type of MAC & 802.11 \\
Type of Network Interface & WirelessPhy \\
Initial Energy & 1 Joule \\
Video sending & MyUDP \\
Video receiving & MyEvalvidSink \\
\hline
\end{tabular}

$$
\text { Avg.Delay }=\frac{1}{n}\left(\sum_{0}^{n} \operatorname{PktRcv}_{\text {TIME }}(n)-\operatorname{PktSent}_{\text {TIME }}(n)\right)
$$

Average Remaining energy (ARE): Relay nodes left with more average remaining energy specify that all the relay nodes are alive for a longer time, which would facilitate to extend the lifetime of the network.

$$
\text { Avg. Re maining Energy }=\frac{\sum_{0}^{n} \text { InitialEnergy }- \text { CurrentEnergy }}{n}
$$

Where,

$n \rightarrow$ Node Count

$\mathrm{Pkt} \rightarrow$ Packet

PktRcv $\rightarrow$ Packet Received

Here, the green color curve represents the ACO-EE mechanism and red color curve represents the EERS mechanism. The number of packets delivered is higher in the ACO-EE technique compared to the EERS mechanism as shown in Figure 2. Due to the FC nodes reduce the maximum packet losses, as a result, increases the received packets rate in the network. 
There are a smaller number of packets lost in the proposed ACO-EE mechanism is shown in Figure 3. This result indicates EERS have the highest data losses in the network. But in ACO-EE the FC nodes capture the loss of packets; thus itreduces the packet losses in the network.

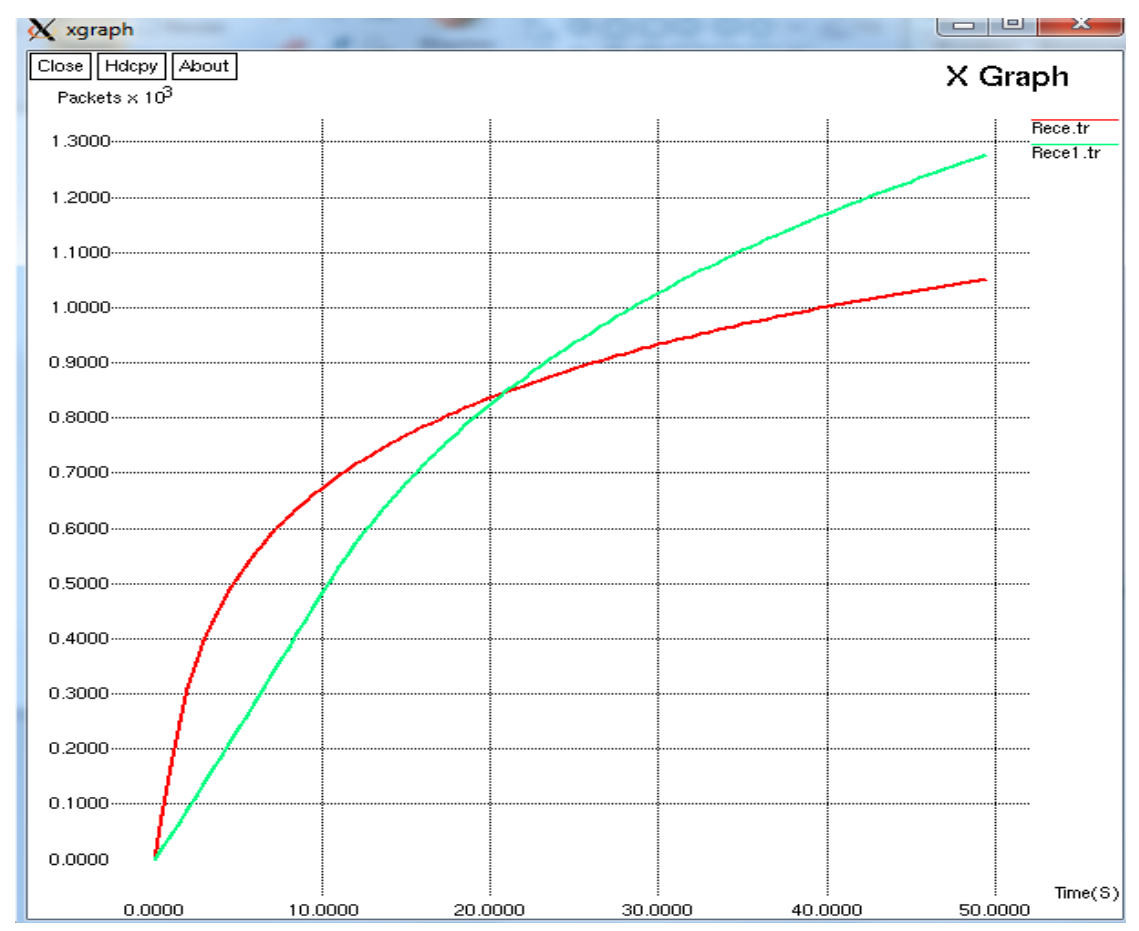

Figure 2. Received Packet Rate of ACO-EE and EERS

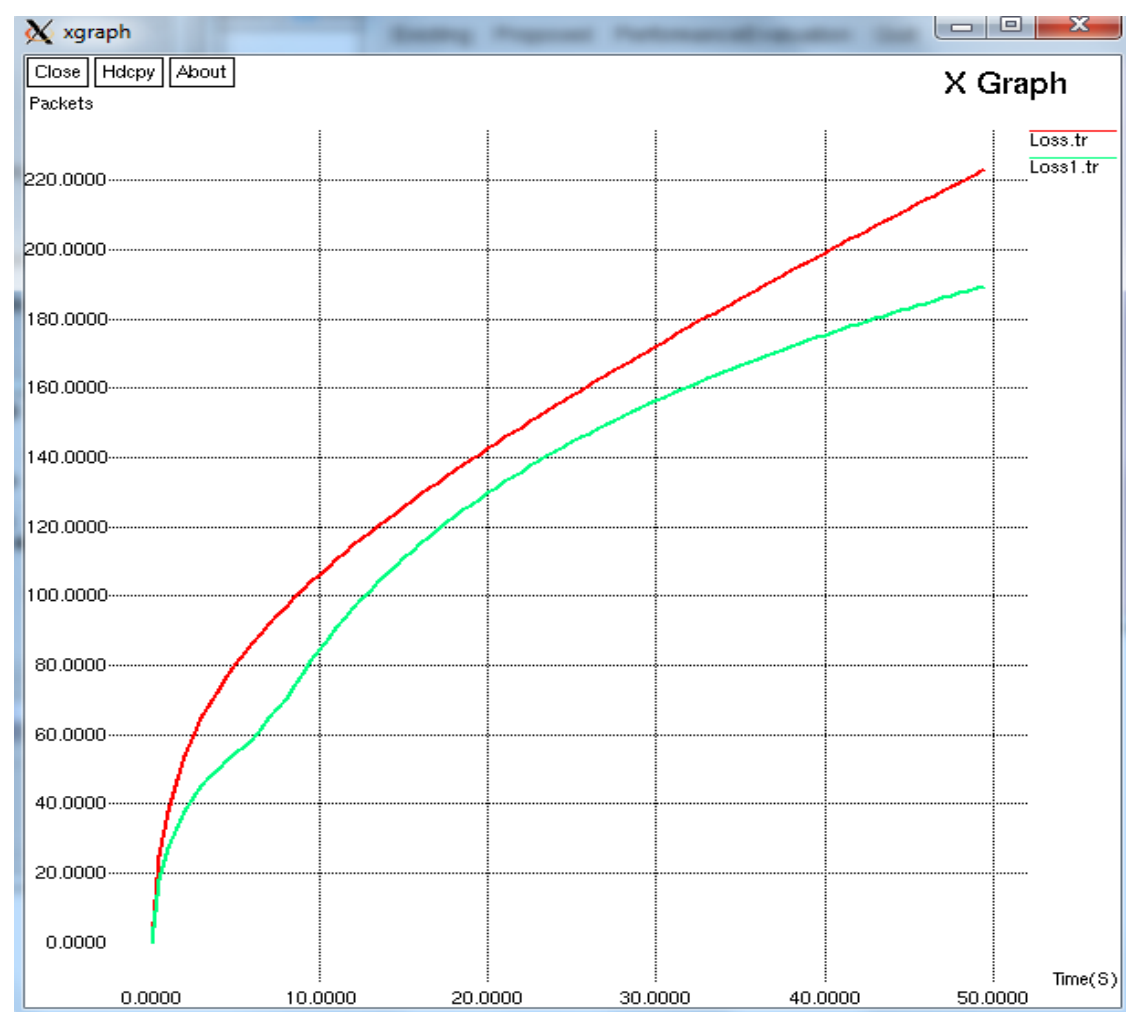

Figure 3. Loss Packet Rate of ACO-EE and EERS 


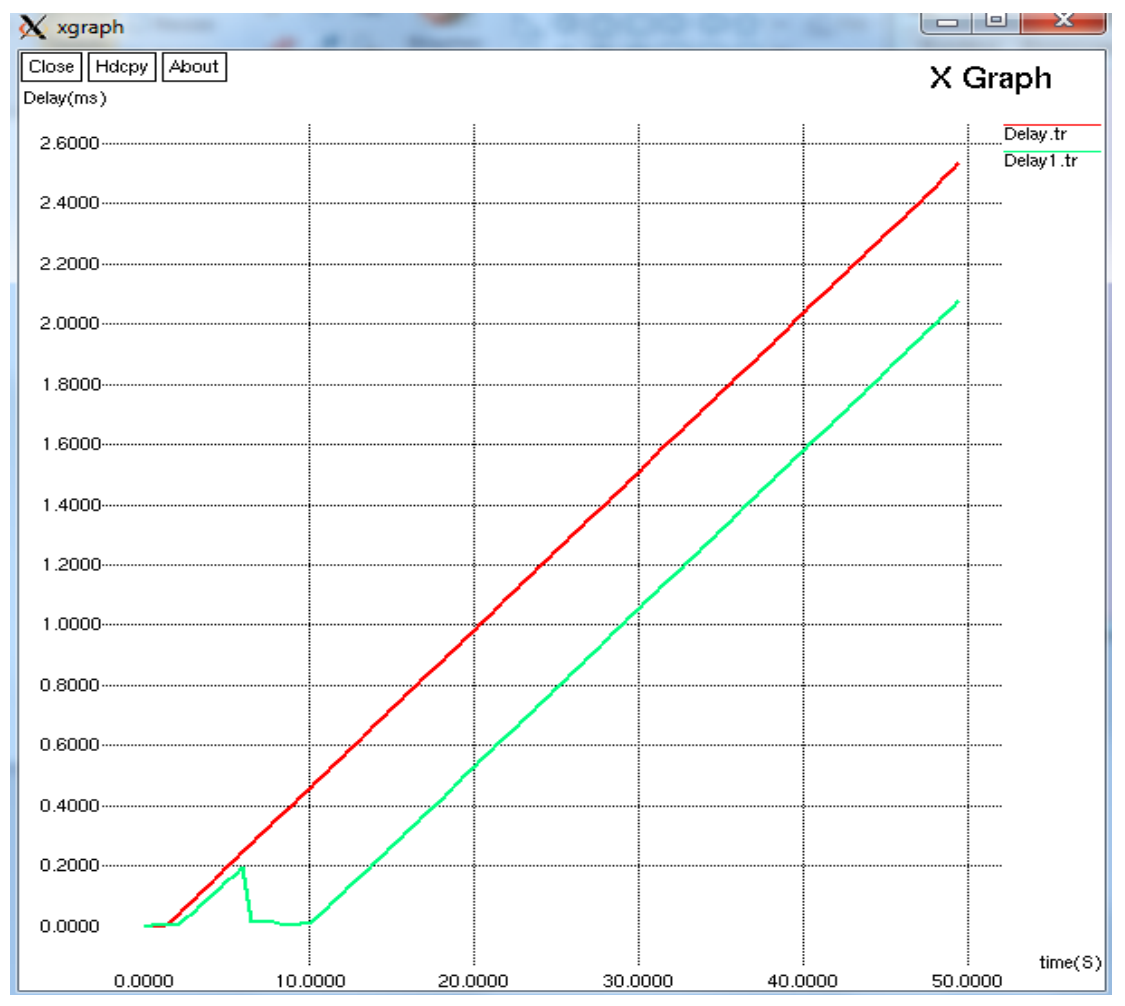

Figure 4. Delay of ACO-EE and EERS

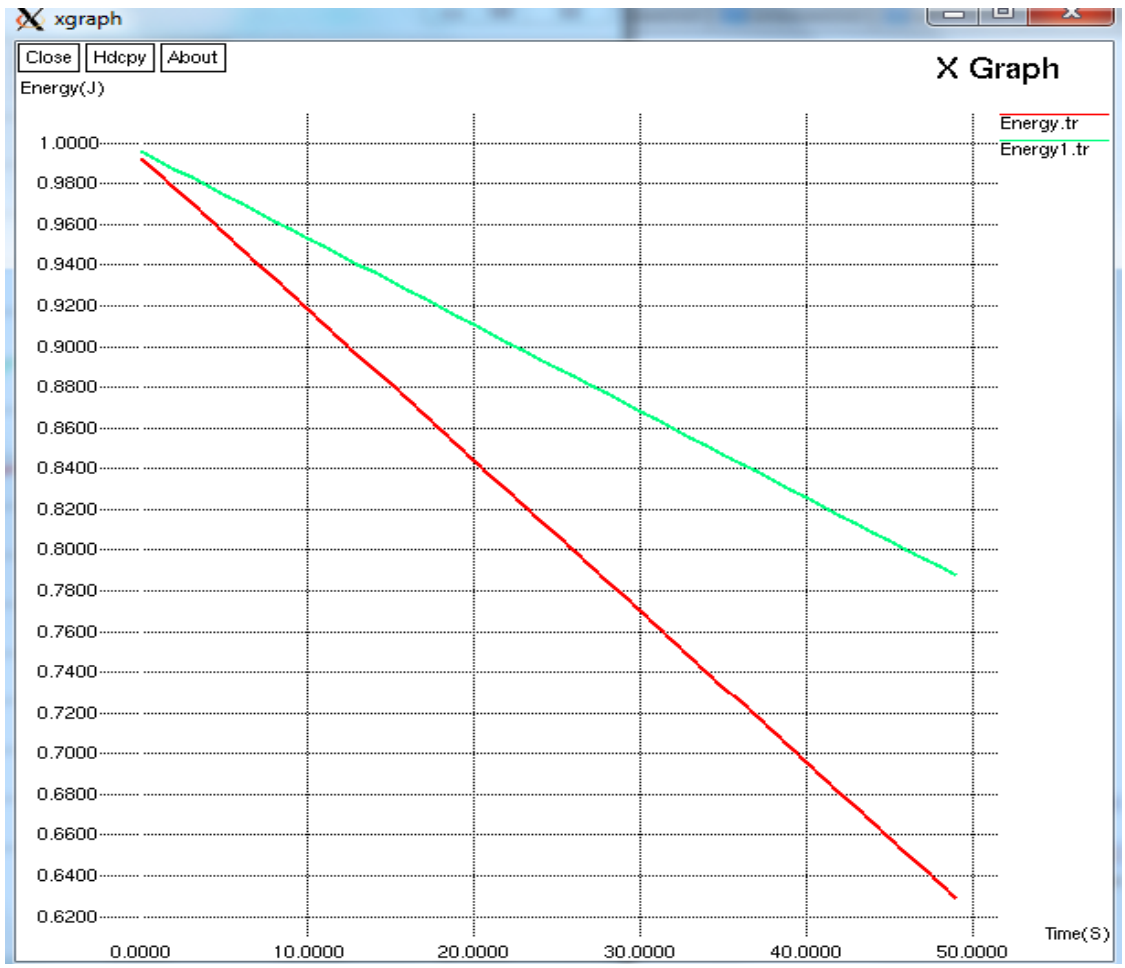

Figure 5. Average Remaining Energy of ACO-EE and EERS

The delay comparison between the ACO-EE and EERS is revealed in Figure 4. The EERS raises the forwarder total transmission time also it does not measure the accurate delay value. As a result, the EERS increases the network delay. But in ACO-EE the forwarding FC nodes reduce the delay. 
Figure 5 demonstrates that the average remaining energy of ACO-EE and EERS scheme. Here, ACO-EE has the highest remaining energy since the relay node, and FC nodes are selected by highest remaining energy. Thus, improve the energy efficiency in the multimedia WMN. The network lifetime is closely related to energy consumption. The higher the network lifetime is, the more effectively the balance of energy will be achieved.

\section{CONCLUSION}

An Ant Colony Optimization based Energy Efficiency for improving opportunistic routing mechanism is proposed in this paper. In, ACO-EE, adaptively chosen's forwarding candidate set nodes from WMNs, and rotates particular time. The optimal transmission distance and remaining energy based energy computation is used to savingnode energy and enhancing the network lifetime. The ant colony optimization algorithm for finding the optimal path and reduce the network delay. The updated ant highest pheromone value node is selected as a FC node. The Node optimal energy, Data communication Time, and hop count energy parameters are used to find the better route in WMN. ACO-EE scheme to maximize the energyefficiency and increase the network lifetimeSimulation analysis shows the ACO-EE minimizing delay and energy efficiency and reducing packet losses compared to the baseline protocol.

\section{REFERENCES}

[1] N. Samian, Z.A. Zukarnain, W.K. Seah, A. Abdullah, Z.M. Hanapi, "Cooperation stimulation mechanisms for wireless multihop networks: A survey”, Journal of Network and Computer Applications, vol. 54, pp. 88-106, 2015.

[2] L.Yuan, C.A. Yuan, and D.S.Huang, "FAACOSE: a fast adaptive ant colony optimization algorithm for detecting SNP epistasis", Complexity, Hindawi, 2017.

[3] S. Kwon, and Shroff, N. B, " Energy-efficient SINR-based routing for multihop wireless networks", IEEE Transactions on Mobile Computing, vol. 8, no. 5, pp. 668-681, 2009.

[4] Y. Wang, X. Y. Li, W. Z. Song, M. Huang, and T. A. Dahlberg, "Energy-efficient localized routing in random multihop wireless networks", IEEE Transactions on parallel and distributed systems, vol. 22, no. 8, pp. 1249-1257, 2011.

[5] D. Zhang, G. Li, K. Zheng, X. Ming, and Z.H. Pan, "An energy-balanced routing method based on forward-aware factor for wireless sensor networks", IEEE transactions on industrial informatics, vol. 10, no.1, pp.766-773, 2014.

[6] S.H. Shivashankar, G.Varaprasad and G. Jayanthi, "Designing energy routing protocol with power consumption optimization in MANET”, IEEE Transactions on Emerging Topics in Computing, vol.2, no. 2, pp. 192-197, 2014.

[7] H. Wang, Y, Chen, and S.Dong, S. "Research on efficient-efficient routing protocol for WSNs based on improved artificial bee colony algorithm”, IET Wireless Sensor Systems, vol. 7, no. 1, pp. 15-20, 2016.

[8] H.H.Kenchannavar, S. Beedakar, and U.P. Kulkarni, U. P "Optimization techniques to improve lifetime of wireless sensor networks: A review”, In 2015 International Conference on Energy Systems and Applications, pp. 446-450, 2015.

[9] A.Taha, R. Alsaqour, M. Uddin, M. Abdelhaq, and T. Saba, "Energy efficient multipath routing protocol for mobile ad-hoc network using the fitness function", IEEE access, vol. 5, pp. 10369-10381, 2017.

[10] Z. Sheng, J. Fan, C.H. Liu, V.C. Leung, X. Liu, K.K. Leung, "Energy-efficient relay selection for cooperative relaying in wireless multimedia networks", IEEE Transactions on Vehicular Technology, vol. 64, no. 3, pp. 1156$1170,2015$.

[11] S.M. Elrabiei, and M.H. Habaebi, "Reliable cooperative multicasting for MBS WiMAX traffic", Computer Networks, vol. 56, no. 11, pp. 2600-2613, 2012.

[12] S.D.Shirkande, R. A. and, R. A.Vatti. "Aco based routing algorithms for ad-hoc network (wsn, manets): A survey", IEEE International Conference on Communication Systems and Network Technologies, pp. 230-235, 2013.

[13] S.Sharma, and R.S.Kushwah." ACO based wireless sensor network routing for energy saving", International Conference on Inventive Communication and Computational Technologies, pp. 150-154,2017.

[14] W.Cai, X, Jin, Y. Zhang, K. Chen, and R.Wang, "ACO based QoS routing algorithm for wireless sensor networks", International conference on ubiquitous intelligence and computing, pp. 419-428, Springer, Berlin, Heidelberg,200

[15] D. Zhang, G. Li, K. Zheng, X.Ming and Z.H. Pan, Z. H. "An energy-balanced routing method based on forwardaware factor for wireless sensor networks", IEEE transactions on industrial informatics, vol. 10, no. 1, pp. 766773, 2014.

[16] J. Li, L. Andrew, C. Foh, M. Zukerman, and H.H. Chen, "Connectivity, coverage and placement in wireless sensor networks", Sensors, vol. 9, no. 10, pp. 7664-7693, 2009. 